\title{
Rückblick aus der Zukunft 50 Jahre danach
}

\section{Peter Marko}

Dr. med., Mitglied FMH

50 Jahre nach der Praxiseröffnung kehre ich zurück in den Ort, den ich einst so beschrieb: «In unserem Dorf praktizieren drei Ärzte: ein Chirurg, ein Internist und ich als Allgemeinpraktiker. Wir vertreten uns gegenseitig während der Ferien und leisten abwechslungsweise Wochenenddienste, auch im Spital, so dass wir über unsere Patienten eine gute Übersicht haben. Unser Gebiet ist zirka $25 \mathrm{~km}$ lang. Ungefähr die Hälfte unserer Patienten sind Bergbauern, die in den kälteren Jahreszeiten ihre Zipfelmützen tragen» [1].

\section{Das Haus, in dem ich die Praxis hatte, ist schöner, stattlicher, aber nach 100 Jahren gibt es dort keine Arztpraxis mehr.}

Jetzt gibt es zwar weniger Bergbauern, aber immer noch viele Bewohner tragen die «gäbigen» Zipfelmützen. Das Haus, in dem ich die Praxis hatte und im ersten Stock wohnte, ist schöner, stattlicher, aber nach 100 Jahren gibt es dort keine Arztpraxis mehr. Der Besitzer, mein Nachfolger, hat mit der Praxistätigkeit, die er nach dem Muster seines Vaters als Chirurg im Spital und praktizierender Arzt führte, aufgehört und im Erdgeschoss die Praxisräume in eine Wohnung für die Familie seiner Tochter umgebaut. Das ist nicht alles, nach der Blütezeit mit mehreren Ärzten, praktiziert im Dorf keiner mehr.

Das schöne Spital, das ein Jahr nach Anfang meiner Praxistätigkeit eröffnet und mehrmals um- und ausgebaut wurde, wollten sie noch vor ein paar Jahren in der Zeit der Liebe zu Grossspitälern schliessen. Nach heftigen Protesten der Einwohner, wie im ganzen Land bei solchen Vorhaben üblich, besann man sich seitens der Behörden eines Besseren. Die Proteste wären vermutlich ins Leere gegangen, aber die verantwortlichen Politiker, die Vorsteher der kantonalen Gesundheitsdirektionen, die es durchsetzen wollten, wurden prompt abgewählt, und ihre Parteien büssten bei den nächsten Wahlen jeweils Stimmen ein. Das zeigte die Stärke der direkten Demokratie.

Darauf wurde nach längeren Diskussionen in der Standes- und allgemeinen Presse ein neues Modell des peripheren Spitals entwickelt, wie es auch hier jetzt bes- tens funktioniert. Es besteht aus einem Kreisssaal, von Hebammen geführt, mit Zimmer für Wöchnerinnen, sowie einer geriatrischen Abteilung. Die Angehörigen der alten Patienten brauchen nicht weit das Tal runter zu fahren, um sie zu besuchen. Ähnlich ist es mit der Demenzabteilung. In der Pflegeabteilung werden Patienten wenn nötig auf die Operationen in den grösseren Spitälern vorbereitet und nachher nachbehandelt, wie auch andere Patienten, die zwar nicht zu Hause betreut, aber auch nicht in einem grösseren, besser ausgerüsteten Spital versorgt werden müssen.

Im Ambulatorium werden kleinere operative Eingriffe, Wundversorgungen vom Personal durchgeführt, anspruchsvolle Endoskopien sowie Ultraschalluntersuchungen jedoch von auswärtigen Spezialisten, die jeweils dafür einreisen. Sie benützen Geräte, die von Zentralspitälern aussortiert wurden.

Die Medikamente für die einzelnen Patienten bereitet ein Roboter vor. Die Spitalapotheke hat auch einen Vorrat an Notfallmedikamenten für die Bevölkerung des Tales. Ihren normalen Bedarf beziehen sie über eine

\section{Die pflegenden Menschen sind nicht ver-} schwunden. Sie springen ein, wenn die Roboter überfordert sind oder jemand sie ablehnt.

Versandapotheke, was für sie und ihre Versicherer günstiger ist. Die Dorfapotheke schloss bereits vor mehreren Jahren ihre Türe. Die Patienten werden auch zum grossen Teil von Robotern gepflegt. Zuerst wagten es nur die Mutigen. Als sie Gefallen an den ruhig summenden und arbeitenden Maschinen fanden, folgten ihnen weitere. Die pflegenden Menschen sind nicht verschwunden. Sie springen ein, wenn die Roboter überfordert sind oder jemand sie ablehnt. Die Qualität der Patientenbetreuung hat enorm zugenommen: Die Patienten werden öfters gepflegt, gewendet, schneller, ohne lange warten zu müssen, gereinigt und ernährt. Das Personal hat mehr Zeit, ihren Ängsten und Sorgen zuzuhören, und da manche dafür besonders geschult wurden, können sie auch in diesem Bereich besser helfen.

Wegen der guten Spracherkennungssysteme und einheitlichen elektronischen Krankengeschichten ver- 


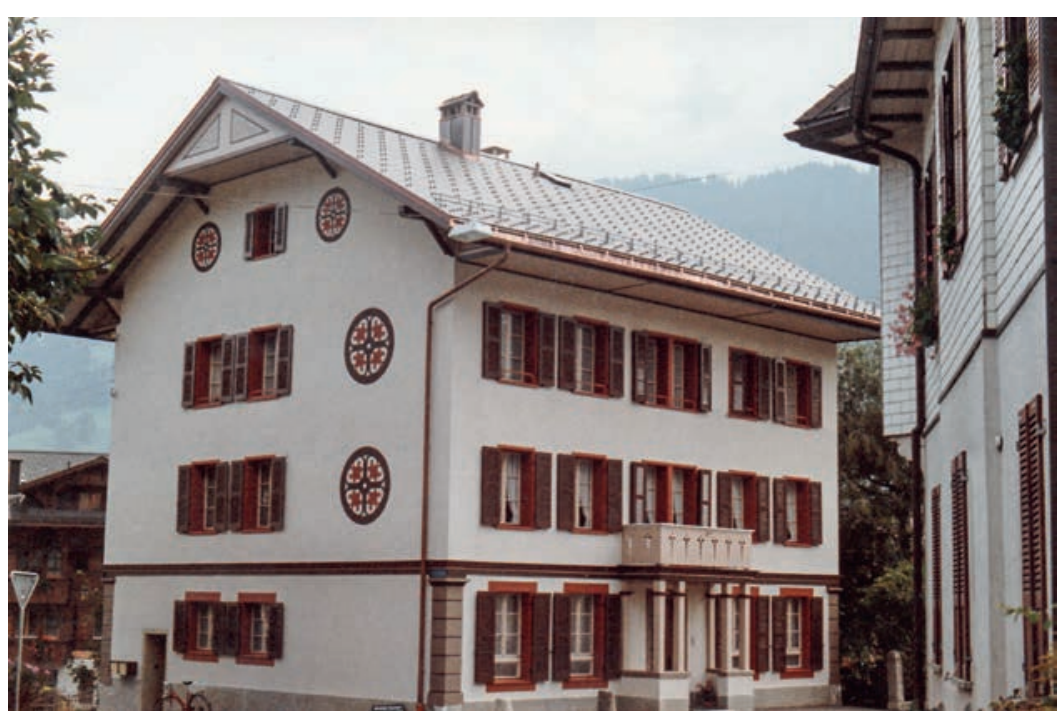

Das ehemalige Praxishaus in Zweisimmen.

minderten sich die ungeliebten Schreibarbeiten, und es gibt mehr Zeit für die Uraufgabe, die Pflege und Betreuung der Patienten. Dank Telemedizin braucht das Spital keine eigenen Ärzte. Als Unterricht für Studenten und junge Assistenten findet einmal pro Woche eine Visite mit einem erfahrenen Arzt, nicht selten einem Professor aus einem Zentrumsspital statt.

In einer Zeit, in der es aus verschiedenen Gründen immer weniger Allgemeinärzte gab, wurde wissenschaftlich nachgewiesen, dass in Gebieten, wo sie vorhanden waren, die Gesundheit besser und die Sterblichkeit tiefer war [2]. Dann steigerte man noch die Bemühungen,

\section{Dank Telemedizin braucht das Spital keine} eigenen Ärzte. Als Unterricht für Studenten und junge Assistenten findet einmal pro Woche eine Visite mit einem erfahrenen Arzt statt.

um mehr Allgemeinärzte zu haben. Es half nicht. In der Not besann man sich, dass eigentlich die Funktion, die Erfüllung der Aufgaben wichtig ist und in der Zeit der künstlichen Intelligenz, diagnostischen und therapeutischen Algorithmen, Telemedizin auch weniger gründlich und weniger lange ausgebildete Personen ohne Hochschulabschluss viele ärztliche Funktionen übernehmen könnten.
Schon seit längerer Zeit übernahm doch nichtakademisches, gut und speziell ausgebildetes Personal viele ehemalige ärztliche Aufgaben wie Diätberatung, Pflege der Wunden bei Diabetikern, Betreuung der Dementen, Versorgung einfacher Wunden. Nebst der Labormedizin spielte die Anästhesiologie eine Vorreiterrolle. Bereits vor mehr als 60 Jahren war es üblich, dass die Patienten von Pflegefachleuten narkotisiert wurden. Dieser Trend verstärkte sich unter dem Titel «Fachüberschreitende Zusammenarbeit». Die immer besseren Geräte für die bildgebenden Untersuchungen ermöglichten ihren Einsatz in der Röntgenologie und bei Endoskopien. 50 Jahre nach der Eröffnung meiner allgemeinärztlichen Praxis werden die Patienten im Tal von besser und von speziell ausgebildetem Spitexpersonal im Ambulatorium des Spitals betreut. Sie besuchen die Patienten selbstverständlich auch zu Hause. Der Vorteil dieser Lösung ist, dass sie meistens ortsgebunden und ortskundig sind und damit weniger ihre Stellen wechseln. Ihre Arbeit ist interessant und sehr begehrt. Das Modell mit speziell ausgebildetem Pflegepersonal, aber ohne Besuche übernahmen auch

Schon seit längerer Zeit übernahm nichtakademisches, gut und speziell ausgebildetes Personal viele ehemalige ärztliche Aufgaben.

grosse zentrale Spitäler, um ambulante Patienten mit kleinen Wunden und banalen Krankheiten zu triagieren und zu versorgen.

Durch diese Entwicklung sowohl im Spital- wie im ambulanten Bereich entschärfte sich in relativ kurzer Zeit der Mangel an Ärzten und Pflegepersonal. Ihre Zuwanderung aus Ländern mit einer schlechteren Belohnung wurde weitgehend aufgehalten. Diese Länder litten mehr darunter und begannen deswegen mit den beschriebenen Änderungen zuerst. Wie alle technischen Fortschritte führten sie zu einer grösseren Effizienz und kürzeren Arbeitszeiten.

\section{Bildnachweis \\ Peter Marko}

\section{Literatur}

1 Marko P. Familienarzt, Familientherapie und ein Fall. Psychosom Med. 1980;9:238-43.

2 Sessums LL, ConwayPH. Saving primary care. JAMA Internal Medicine. 2017;177(11):1561-2. 